\title{
Expert Knowledge Guided Segmentation System for Brain MRI
}

\author{
Alain Pitiot ${ }^{1,2}$, Hervé Delingette ${ }^{1}$, Nicholas Ayache ${ }^{1}$, and Paul M. Thompson ${ }^{2}$ \\ 1 Epidaure, INRIA, 2004 route des lucioles BP 93, 06902 Sophia-Antipolis, France, \\ \{alain.pitiot, herve.delingette, nicholas.ayache\}@sophia.inria.fr \\ 2 LONI, UCLA School of Medicine, Los Angeles, CA 90095, USA \\ thompson@loni.ucla.edu
}

\begin{abstract}
This paper presents a fully automated segmentation method for extracting a variety of anatomical structures in magnetic resonance images (MRI). We have developed a segmentation system where maximum use is made of the available medical expertise, either in the form of implicit knowledge or of explicit information. A series of deformable templates (simplex meshes), initialized via the non-linear registration of a reference segmented MRI, are evolved in a rule-controlled framework and subject to various constraints, so as to maximize the achieved match over the target structures. Segmentation results on brain MRIs are discussed and compared against manual delineations.
\end{abstract}

\section{Introduction}

Effective segmentation of anatomical structures in possibly complex MRI proves to be especially challenging, given the wide variety of shapes and intensities a structure can present. Yet, image segmentation calls for high precision since the quality of the subsequent studies often depends on how accurately the various structures in the image can be identified. Similar needs for automated segmentation arise when building brain atlases (see [1] for a review). These atlases can still be rather tedious to build, as many components typically have to be interactively outlined. Thus, automated segmentation systems can be powerful tools to help in drawing consistent analyses from a number of images or collect statistical information on anatomical variability.

\subsection{Prior Work}

Several segmentation methods have been proposed in the literature to extract anatomical structures, using an array of feature descriptors and shape models. In view of the complex nature of the problem, deformable templates seem the weapon of choice. First, they can adequately handle the discontinuities and irregularities that sampling artifacts or noise may induce along the boundaries of the target structures. Their compactness enables a wide variety of shapes to be described while minimizing the overall number of parameters or masking them 
behind a small and easily manageable set of physical principles. They also provide an analytical model of the structure once segmented, which facilitates its subsequent analysis. Finally, a priori knowledge on the shape, location, or appearance of the target structure can be used to guide the deformation process. Reviews of various deformable template techniques can be found in [2] and [3]. Among others, Staib and Duncan [4] used elliptic Fourier decomposition to encode the structure boundaries within a probabilistic deformable model. Active appearance models 5] incorporate both a statistical model of the shape of the target structure and a description of the statistical distribution of its gray-level intensities. Pizer at al. 6] used medial representations (chains of medial atoms with associated information: width, tangent plane, ...) to segment anatomical structures. Intensity profiles can also provide an efficient means to introduce $a$ priori knowledge. Brejl and Sonka 7] for instance used a border appearance model to automatically design cost functions that serve as a basis for the segmentation criteria for edge-based segmentation approaches.

Most of these approaches fall in the implicit knowledge category: from a learning set of a priori segmented instances of an anatomical structure, they have to automatically discover the relationships and functional dependencies of the various parameters of the model. However, explicit information about the target structures is often available, in the form of medical expertise. For instance, the relative positions of most of the deep grey nuclei is fairly constant across individuals, anatomical structures should not to intersect, etc. From these observations, a series of rules can be derived to better drive the segmentation process. Broadly speaking, explicit knowledge approaches can be regarded as a special case of implicit knowledge algorithms where the additional medical expertise provides short cuts in the search for the target structure. Many expert systems have been detailed in the literature [8,9,10] where rules model a variety of features: position, shape, pixel-level properties, textures, etc.

\subsection{Proposed Approach}

This paper addresses the problem of retrieving the boundaries of a selection of target anatomical structures in a 3-D MR image. We approach the issue of boundary finding as a process of fitting a series of deformable templates to the contours of these structures. We chose simplex meshes [1] to model the templates, owing to their fairly simple geometry, which makes it easier to incorporate internal and external constraints. We have focused on devising a segmentation system where maximum use is made of the available medical expertise, either in the form of implicit knowledge (the shape of the structures, their appearance, ...) or of explicit information (the relative distance between structures, the rules for the feedback loops, ...), implemented as constraints on the meshes.

Figure 1illustrates the proposed framework. Non-linear registration of a reference MRI serves to initialize a series of simplex meshes (one mesh per target structure) and makes the approach more robust (see section 2.2). Each simplex mesh is then iteratively modified to minimize a hybrid local/global energy which incorporates an internal regularization energy, an external term which couples 


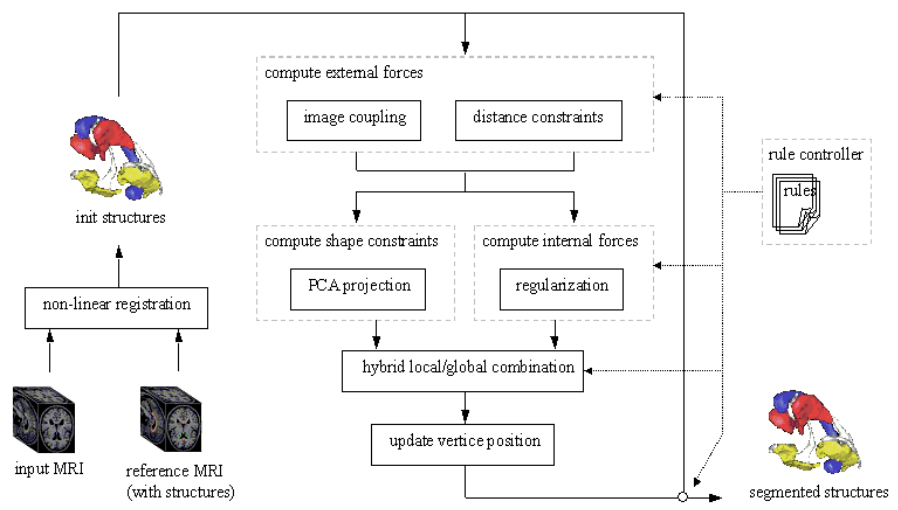

Fig. 1. Overview of the proposed segmentation system

the models to the underlying image features and a global shape-constrained term. Those meshes evolve in parallel, within a rule-controlled framework whose purpose is to maximize the achieved match over each structure while respecting distance, position, etc. constraints (derived from medical expertise).

We detail in the following section 2 the various components of our segmentation system before presenting some qualitative and quantitative results.

\section{Knowledge-Driven Segmentation System}

\subsection{Deformation Model}

Simplex meshes [11] were selected to model the evolving deformable templates. A simplex mesh is a discrete model representation (a set of vertices and edges) with prescribed vertex connectivity. To encode the structure surfaces, we use 2simplex meshes: each vertex is then connected to exactly three neighbors. This inherent geometric simplicity greatly eases the imposition of constraints (internal or external) to bias the segmentation process. Additionally, "zones" (subsets of vertices) can be defined on the simplex meshes to further specify the constraints.

More formally, we define the input MR image by its intensity at each point: $I: \Omega \subset \mathbb{R}^{3} \rightarrow \mathbb{R}$, and a 2-simplex mesh as a set of points $\Pi=\left\{P_{i} \in \mathbb{R}^{3}\right\}_{i=1}^{N}$ along with its associated connectivity matrix (which is constant in our case since we do not allow topological changes). The algorithm's goal is then to find in $I$ a pictorial object whose overall boundary fits that of $\Pi$. To guide the deformation process and drive the template towards the required object boundary, we introduce a compound energy $E$ whose minimum we aim to determine. Classically, $E$ is made up of two terms: an internal (or regularization) energy $E_{\text {int }}$ which characterizes the possible template deformations, and an external energy $E_{\text {ext }}$ which couples the template to the image: $E=\alpha \cdot E_{\text {int }}+\beta \cdot E_{\text {ext }}$, with $\alpha, \beta \in \mathbb{R}$. Within a Newtonian framework, we get the following iterative point updating 

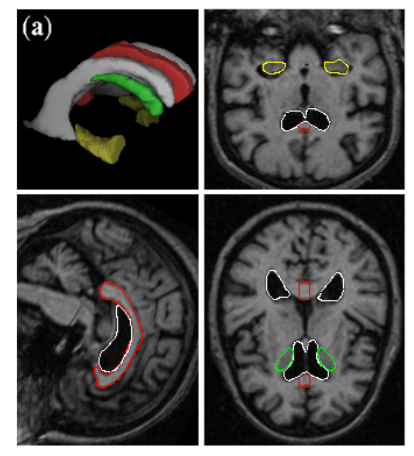
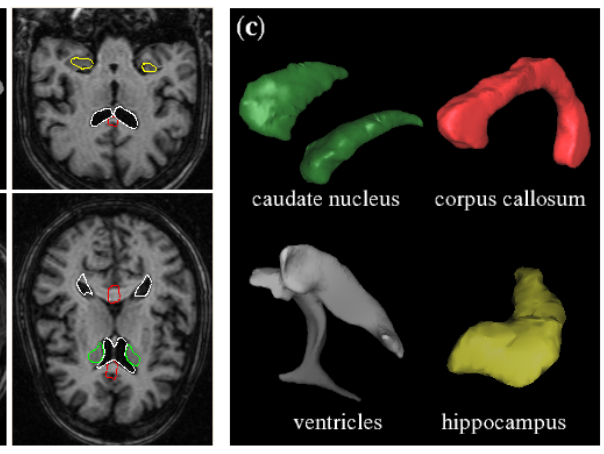

Fig. 2. (a) Reference MRI with superimposed manually delineated structures; (b) reference MRI registered to an input MRI and transformed structures; (c) a few segmented structures (caudate nucleus, corpus callosum, lateral ventricles and hippocampus)

procedure: $\Pi^{t+1}=\Pi^{t}+(1-\delta)\left(\Pi^{t}-\Pi^{t-1}\right)+\alpha \cdot f_{\text {int }}\left(\Pi^{t}\right)+\beta \cdot f_{\text {ext }}\left(\Pi^{t}\right)$ where $\delta$ is a damping coefficient $(0<\delta<1)$.

A number of external forces are available. They are either based on the gradient of the input image, on a smoothed version of its associated edge-image, or on intensity profiles, etc. We use the distance to the closest strong gradient in the underlying image as it exhibited the best trade-off between precision and robustness [11. As a internal force, we average those deformations over a spherical neighborhood (whose size is an additional parameter).

\subsection{Initialization}

Once we have reduced the segmentation problem to an energy minimization task, we face a multi-modal, non-linear and possibly discontinous function of many variables. Given the size and non-convexity of the solution space, most minimization technique would only lead to weak sub-optimal solutions (where the deformation model adapts to mere noise or decoys or maybe only follows parts of the desired boundaries) if the search space were not drastically reduced by assuming that a good approximation to the solution is available. This could be either in the form of a set of pose parameters (position, orientation, scale) or shape descriptors (possibly those of the mean shape model).

Various approaches have been presented in the literature to overcome this robustness issue. In [12] for instance, a coarse to fine strategy, the Graduated Non-Convexity Algorithm, is implemented, where a scalar parameter controls the amount of "local" convexity. Alternatively, the templates can be initialized at a number of locations and evolved in sequence: the deformed template with the best final match is then selected. In [13], a hybrid evolutionary algorithm controls a family of deformable templates that are evolved simultaneously and explore the search space in a robust fashion. Here, we use nonlinear registration to initialize the templates reasonably close to their expected positions. 
An MRI brain dataset was selected for its "standard" appearance (the reference MRI), and we carefully segmented in it the target structures (see Figure 2]a). Given an input MRI to be processed, we first register the reference MRI to it with the MAMAN 14 algorithm (non-linear registration with an elastic prior). The obtained transform is then applied to the meshes segmented in the atlas. Those transformed meshes serves as initial guesses for the segmentation of the target structures (Figure 2.b). Note that we also envisioned using an iconic atlas 15. (the average of a number of MRIs linearly or nonlinearly registered to themselves) as a reference MRI. However, the nonlinear registration of the atlas to the input MRI provided inferior initializations as some of the strong features used by MAMAN (edges, ridges, ...) were not as clearly defined in the true atlas than in the "average-looking" MRI.

\subsection{Knowledge-Based Constraints}

We have introduced a number of constraints (either in the form of new energy terms or of a series of rules) to bias the segmentation process towards shapes that are deemed valid with respect to the a priori medical knowledge we have gathered on the target structures.

Shape constraints. In spite of the large variability of brain structures, the notion of biological shape seems reasonably well explained by a statistical description over a large population: the so-called "shape model". A deformable template is then not only constrained by the number of degrees of freedom imposed by its geometric representation, but also in that it must be a valid (or close to valid) instance of the shape model. Given, for each target anatomical structure, a series of pre-segmented instances, we first reparameterize and align them (see 13] for details) before computing the principal modes of variation following Cootes' Principal Component Analysis (PCA) methodology [5]: those modes correspond to the eigenvectors associated with the largest eigenvalues of the variance/covariance matrix computed over the homologous points of the reparameterized and aligned instances.

Once we have a shape model for each structure, the deformable templates must be constrained accordingly. In [5], the pose and shape parameters of the templates are adjusted by projecting the local deformation induced by the external energy onto the shape space: this limits the range of possible shapes to the only valid ones with respect to the shape model. Let $d \Pi_{\text {ext }}^{t}=f_{\text {ext }}\left(\Pi^{t}\right)-\Pi^{t}$ be the displacement induced by the external forces. Let $\bar{S}$ be the mean shape computed for the target structure, and $Q=\left\{q_{1}, \ldots, q_{m}\right\}$ its $m$ first eigenmodes. The shape-constrained deformation is written: $d \hat{\Pi}_{\text {ext }}^{t}=\sum_{i=1}^{m}\left\langle\Pi^{t}+d \Pi_{\text {ext }}^{t}-\bar{S}, q_{i}\right\rangle \cdot q_{i}$. Alternatively, in [13] a twolevel hybrid evolutionary algorithm intertwined shape-model constrained steps and free-deformation iterations, yielding a more flexible deformation framework. Here we use a hybrid local/global scheme [16] where deformations are regularized through a combination of global (shape constrained) and local (external) 
forces. The point updating rule becomes: $\Pi^{t+1}=\Pi^{t}+(1-\delta)\left(\Pi^{t}-\Pi^{t-1}\right)+$ $\lambda .\left\{\alpha \cdot f_{\text {int }}\left(\Pi^{t}\right)+\beta \cdot f_{\text {ext }}\left(\Pi^{t}\right)\right\}+(1-\lambda) \cdot\left\{d \hat{\Pi}_{\text {ext }}^{t}\right\}$, where $\lambda$ is the "locality" parameter which controls the contribution of the global shape-model constraint.

Distance constraints. In [17], fuzzy logic was used to express distance and positional relationships between structures. Here we choose distance maps, owing to their ability to model more precise constraints (to guarantee non intersection for instance). Given a deformable template $\Pi_{0}^{t}$, we wish to impose on it a distance constraint with respect to template $\Pi_{1}^{t}$. We first compute the distance map $D_{1}^{t}$ associated with a discrete sampling of $\Pi_{1}^{t}$. At each vertex $P_{i}^{t}$ of $\Pi_{0}^{t}$, we can then compute a "distance force" $f_{\text {dist }}$ whose magnitude depends on the value of the distance map at the considered vertex. Two types of constraints can be applied: we can either wish the force to attract the vertex, along the direction of the gradient of the distance map, up to an exact distance $d_{\text {target }}$ of the target mesh, or only enforce that it should remain at distance inferior or superior to $d_{\text {target }}$ (to prevent intersections between structures for instance). We get: $f_{\text {dist }}\left(P_{i}^{t}\right)=-\frac{\nabla D_{1}^{t}\left(P_{i}^{t}\right)}{\left\|\nabla D_{1}^{t}\left(P_{i}^{t}\right)\right\|} \cdot\left\{D_{1}^{t}\left(P_{i}^{t}\right)-d_{\text {target }}\right\}$. Note that the forces can be applied to a subset of the mesh vertices (so-called "zones") to enforce more local constraints.

Segmentation rules. In view of the complexity of the segmentation task, choosing a value for the various scalar parameters that control the contributions of the above mentioned energies is not a trivial matter. Similarly, there is not a single prescription for the amount of regularization to apply to the templates. Instead of setting a priori sub-optimal values, those parameters could evolve dynamically along with the deformation process. Additionally, instead of segmenting the structures independently and running the risk of them intersecting one another, better segmentation results could be obtained by evolving the templates in parallel while controlling their inter-relationships.

We have consequently developped a rule-based system to manage these hyperparameters. For each target structure, a set of rules was developped that took into account recommendations from clinicians as well as low-level image observations. Due to a lack of space, we only illustrate a few of these rules here.

rule a: Ventricles are segmented first (as they are quite contrasted in the T1weighted MRIs).

rule b: Then, distance constraints (non-intersection condition) help segmenting the caudate nuclei and the corpus callosum.

rule c: For each deformation process, we progressively refine the image gradient used in computing the external forces to garantee deformation at early stages and later ensure a precise delineation (dynamic coarse-to-fine approach).

rule d: Similarly, the locality parameter is initially set to 0.0 and then slowly increased as the deforming template approaches the borders of the target structure. 
A number of feedback loops were also developed to monitor the possible mistakes during the deformation process and react adequately. For instance, one way to ensure that a deformable template does not "leak" outside of the correct boundary is to check that its distance (mean distance averaged over all vertices) to its associated shape-constrained projection stays reasonable at early stages in the deformation process.

\section{Segmentation Results}

We present here some quantitative results for 4 structures (corpus callosum, caudate nucleus, ventricles and hippocampus) for which 20 manually segmented instances are available.

The accuracy was evaluated following the methodology presented in [18. We used as error metrics the partial maximum surface distance (95\% quantile of the model-to-manual and symmetrized Hausdorff distances) and the mean absolute surface distance. Table 1 reports these 3 measures for all 4 structures, averaged over the 20 test instances (different from training instances). Segmentation of caudate and callosum was good and further improved with the use of shape and distance constraints. A few odd-looking caudates (far from the mean shape) worsened the performances when PCA was added. The less accurate segmentation of the ventricles is explained by the inability of our deformable templates to reach as far as the apex of the inferior horns as they would have to go through partial volume effect voxels. Yet the model-to-manual maximum distances were good, since our approach correctly segmented the parts of the ventricles that were "reachable". The self-correcting rules were overall particularly effective, most especially in reducing the maximal errors. However, poor constrast and noise hampered the hippocampus deformable templates. PCA constraints could not help either as the mean shape models were computed after a delineation protocol which artificially limited the extent of the tail of the hippocampus. The use of texture filtering [13] should increase the overall performances.

\section{Conclusion}

We have presented a general framework for automated segmentation of anatomical structures in brain MRI. A hybrid combination of external and internal energies, modeling a variety of aspects of prior medical knowledge, drives a series of deformable template towards the boundaries of these target structures. A number of explicit rules, also derived from medical expertise, further increases the overall accuracy and robustness of the method. The validity of our approach was demonstrated on 4 structures. We plan to use this framework to tackle the segmentation of more structures, and study at greater length the multivariate relations between the various parameters of the deformation scheme and how they affect the accuracy of the match. In particular, additional feedback loops can be devised to tackle the segmentation of difficult images where robustness becomes a more pressing issue. 
Table 1. Segmentation results

\begin{tabular}{|c|c||c|c|c|c|}
\hline system & distance $(\mathrm{mm})$ & corpus callosum & ventricles & caudate nucl. & hippocampus \\
\hline \hline basic & mean & 1.3 & 5.6 & 4.2 & 3.5 \\
framework & $95 \%$ Hausdorff & 2.1 & 4.5 & 4.7 & 8.2 \\
& $95 \%$ symmetrized & 2.2 & 7.5 & 4.8 & 8.8 \\
\hline \multirow{3}{*}{+ PCA } & mean & 1.4 & 5.2 & 3.8 & 4.6 \\
& $95 \%$ Hausdorff & 2.4 & 4.6 & 5.5 & 9.2 \\
& $95 \%$ symmetrized & 2.4 & 8.4 & 5.6 & 10.6 \\
\hline + distance & mean & N/A & 5.0 & 1.5 & N/A \\
constraints & $95 \%$ Hausdorff & N/A & 4.0 & 3.0 & N/A \\
& $95 \%$ symmetrized & N/A & 6.2 & 3.2 & N/A \\
\hline + feedback & mean & 1.2 & N/A & 1.6 & 4.8 \\
rules & $95 \%$ Hausdorff & 2.0 & N/A & 2.4 & 8.9 \\
& $95 \%$ symmetrized & 2.0 & N/A & 2.5 & 9.7 \\
\hline
\end{tabular}

\section{References}

1. Subsol, G.: A Scheme for Automatically building 3D Morphometric Anatomical Atlases Based on Feature Lines: a List of References. DIKU International Summer School98 Shape Variation (1998)

2. McInerney, T., Terzopoulos, D.: Deformable Models in Medical Image Analysis: a Survey. Medical Image Analysis 1 (1996) 91-108

3. Montagnat, J., Delingette, H., Ayache, N.: A review of deformable surfaces: topology, geometry and deformation. Image and Vision Computing 19 (2001) 1023-1040

4. Staib, L., Duncan, J.: Boundary Finding with Parametrically Deformable Models. IEEE Trans. on PAMI 14 (1992) 1061-1075

5. Cootes, T., Edwards, G., Taylor, C.: Active Appearance Models. In: Proc. of the ECCV. (1998) 484-498

6. Pizer, S., Fritsch, D., Yushkevich, P., Johnson, V., Chaney, E.: Segmentation, Registration, and Measurement of Shape Variation via Image Object Shape. IEEE TMI 10 (1999) 851-865

7. Brejl, M., Sonka, M.: Object Localization and Border Detection Criteria Design in Edge-Based Image Segmentation: Automated Learning from Exemples. IEEE TMI 19 (2000) 973-985

8. Brown, M., Wilson, L., Doust, B., Gill, R., Sun, C.: Knowledge-based Method for Segmentation and Analysis of Lung Boundaries in Chest X-ray Images. Computerized Medical Imaging and Graphics 22 (1998) 463-477

9. Matesin, M., Loncaric, S., Petravic, D.: A Rule-Based Approach to Stroke Lesion Analysis from CT Brain Images. In: Proc. of Second International Symposium on Image and Signal Processing and Analysis. (2001) 219-223

10. Ardizzone, E., Peri, D., Pirrone, R., Palma, A., Peri, G.: A Knowledge based Approach to Intelligent Data Analysis of Medical Images. In: Proc. of IDAMAP. (2001) 224-237

11. Delingette, H.: General object reconstruction based on simplex meshes. International Journal of Computer Vision 32 (1999) 111-146

12. Blake, A., Zisserman, A.: Visual Reconstruction. MIT Press (1987)

13. Pitiot, A., Toga, A., Thompson, P.: Adaptive Elastic Segmentation of Brain MRI via Shape-Model-Guided Evolutionary Programming. IEEE TMI 21 (2002) 910923 
14. Cachier, P., Bardinet, E., Dormont, D., Pennec, X., Ayache, N.: Iconic Feature Based Nonrigid Registration: The PASHA Algorithm. CVIU — Special Issue on Nonrigid Registration (2003) In Press.

15. Collins, D.L., Zijdenbos, A.P., Paus, T., Evans, A.C.: Use of registration for cohort studies. In Hajnal, J., Hawkes, D., Hill, D., eds.: Medical Image Registration. (2003)

16. Montagnat, J., Delingette, H.: Globally constrained deformable models for 3D object reconstruction. Signal Processing 71 (1998) 173-186

17. Barra, V., Boire, J.: Automatic Segmentation of Subcortical Brain Structures in MR Images Using Information Fusion. IEEE TMI 20 (2001) 549-558

18. Gerig, G., Jomier, M., Chakos, M.: Valmet: a new validation tool for assessing and improving 3D object segmentation. In: Proc of MICCAI. (2001) 516-528 